\title{
The effect of parent-administered infant massage on the developmental outcomes of premature infants
}

\author{
*Megan Campbell; BSc Occ. Ther. (WITS), MSc Occ. Ther. (WITS). https://orcid.org/0000-000 I-8292-8774 \\ Chief of Paediatric Occupational Therapy, Chris Hani Baragwanath Academic Hospital.
}

\author{
Lizelle Jacobs; B.Occ. Ther. (UP), MSc Occ. Ther. (Brunel University, London), PhD Occ. Ther. (WITS). \\ http://orcid.org/0000-0002-5373-2I55 \\ Lecturer, University of the Witwatersrand Faculty of Health Sciences, School of Therapeutic Sciences, Department of \\ Occupational Therapy.
}

Introduction: Prematurely born infants often have developmental delays in multiple areas when compared to infants born at term. Neurodevelopmental supportive care techniques such as Kangaroo Mother Care or infant massage have the potential to affect the infant's developmental outcome. Evidence supports the benefits of parent-administered infant massage in terms of the premature infants' growth, weight gain and length of hospitalisation. However, the effect of infant massage on other aspects of development has not yet been established.

Method: A randomised controlled trial was used to investigate the developmental milestones of infants who received parent-administered infant massage. The Peabody Developmental Motor Scales Second Edition (PDMS-2) test was used to assess infants at birth and again at four to six months (actual age) along with the Bayley Scales of Infant and Toddler Development Third Edition (BSID-III).

Results: Positive clinical effect sizes were shown in the massaged group in the Cognitive, Reflexes and Visual-Motor Integration subtests. There was also a strong correlation between frequency of massage and the social-emotional development of the massaged infants. Facilitators and barriers, in performing the infant massage programme, were established.

Conclusion: This study supports the use of parent-administered infant massage for premature infants older than 32 weeks gestation to assist with the attainment of their developmental milestones as well as the parent-infant bonding process.

Key words: Infant massage, premature infant, developmental delay, parental involvement

\section{INTRODUCTION}

Premature infants display different capabilities to those born at fullterm, as the premature infant has a period of foetal development outside the supportive environment of the uterus'. The capabilities of the premature infant are therefore impacted as the infant undergoes stressful and sometimes painful experiences before reaching gestational age ${ }^{1,2}$. A premature infant's underdeveloped sensory system, can be overloaded with stimuli during medical procedures, resulting in them responding negatively to the same stimulus that an infant, born at term, responds positively to ${ }^{1,2}$. The developmental trajectories of premature and full-term infants often differ significantly. Infants born prematurely have been shown to have delayed developmental milestones when compared to term infants ${ }^{3,4}$. In a 2014 study on premature infants with low birth weights, it was found that at a corrected age of 18-24 months, children displayed developmental delays in multiple areas such as motor skills, cognition and language ${ }^{5}$. Furthermore, infants who are born prematurely or extremely prematurely, are at risk for later visual-motor integration and higher-order visual problems which impact on school-related tasks ${ }^{6-9}$. Traditionally, occupational therapy offered to premature infants addressed specific deficits such as abnormal muscle tone, decreased range of motion at specific joints or a developmental delay which could have multiple causes ${ }^{10}$.

Neurodevelopmental supportive care (NDSC) is currently the fundamental multidisciplinary approach practised in neonatal units $^{10,11}$. NDSC or a neuroprotective strategy is any intervention that supports the development of a premature infant's brain and neuronal wiring ${ }^{12,13}$. Every interaction that a premature infant has with a caregiver (medical staff or parents) has the potential to affect the infant's developmental outcome ${ }^{14}$. Therefore, therapeutic care of premature infants must involve not only the infant, but also the caregivers to ensure carry-over and success ${ }^{1,10,15}$. Positive touch, one component of NDSC, includes techniques such as Kangaroo Mother Care (KMC), promotion of calming strategies during medical or care procedures, teaching parents massage techniques and positioning of the infant. Infant massage has been shown to have multiple benefits, especially in populations of premature infants ${ }^{16-19}$. The aim of this research was to determine the effect of parent-administered premature infant massage on the acquisition of developmental milestones at four to six months actual age.

\section{LITERATURE REVIEW}

Touch is the first sense a fetus will develop ${ }^{20}$ and at approximately 12 weeks, a fetus is able to detect touch throughout its body ${ }^{18}$. The touch experienced by a fetus is deep pressure or proprioceptive input which enables the fetus to develop a sense of its body and its boundaries $^{18}$. The positive touch a fetus experiences helps start the formation of a bond between the mother and the fetus ${ }^{20}$. Once 
the infant is born, touch input can be used as a calming strategy by the caregiver of the child ${ }^{18}$.

Touch is crucial to any new born infant's development, attachment and well-being ${ }^{18,21}$. A lack of touch can lead to stunting of an infant's physical and emotional development, limitations in their formation of secure attachments, as well as possible failure to thrive later in life ${ }^{18,21,22}$. Positive forms of touch such as the positive touch technique or massage can negate the negative effects of painful procedures and assist a preterm infant to remain in a regulated state $^{23}$. A well-regulated state is where infants are most likely to learn new skills and is therefore essential for development ${ }^{23}$. Infant massage is a form of positive touch that can be used in premature infants to assist in central nervous system development ${ }^{14}$. Central nervous system development will in turn allow the infant to selfregulate and process information with improved efficiency, which can improve the infant's occupational participation. The occupations that are expected of the new-born include: social play, feeding, exploration and procuring'.

McClure was a pioneer adapting traditional massage, specifically from the Indian culture, to a practice that is now widely used in most cultures ${ }^{22}$. While working at an orphanage in India, McClure became aware that Indian mothers regularly massaged their infants ${ }^{22}$. She noted that children who had been massaged were warm and playful and willing to accept responsibility, despite the poverty-stricken circumstances in which they grew up ${ }^{22}$. She took massage strokes from Indian and Swedish massage methods and combined these with techniques she had employed as a yoga instructor $^{22}$. McClure developed the massage techniques for infants that is currently taught globally by the International Association of Infant Massage (IAIM) to their instructors ${ }^{22}$. Infant massage instructors trained by the IAIM do not necessarily need to be therapists or have a medical background in order to undergo the training. Infant massage instructors are however, taught to read the infant's coping signals and be able to explain these to parents to avoid any detrimental effects on the infant. During the training, instructors are taught to provide instruction to parents on the practise of infant massage. At no time does the instructor massage the infant, but they rather provide instruction and support to the parents in this process. Westernised infant massage has recently become popular due to the reported benefits for the parent and the infant ${ }^{19}$.

The benefits of infant massage in Western countries include higher bone density in premature infants, improved weight gain, pain reduction, increased feeding tolerance, better sleeping patterns and improved parent-child attachment and bonding 16, 18, 19, 24 . Weight gain in particicular is important as premature infants who are medically stable often have to remain in hospital until they have gained sufficient weight to be discharged home safely ${ }^{16}$. In a 2007 study, premature infants who were massaged daily were shown to gain more weight than those who did not receive massage intervention ${ }^{24}$. Similarly, in a study where mothers, who were human immunodeficiency virus (HIV) positive, were taught to massage their infants daily, massaged infants also put on more weight than their counterparts who were not massaged ${ }^{25}$. These above-mentioned benefits of infant massage often decrease the length of hospitalisation of infants which has a favourable financial implication for hospitals and health care funders ${ }^{16}$.

Infant massage not only benefits the infants but also the parents providing the massage and it has been shown to reduce anxiety in mothers of premature infants, increase their confidence in their parenting ability and assist them with the transition into their new role $^{26}$. A 20 I I study suggested that increasing a mother's sensitiv- ity to her infant's cues as part of a massage training programme, may have a positive effect on the disposition of the infant and the parent-infant relationship ${ }^{25}$. Furthermore, infant massage has been reported to improve interactions between infants and their mothers in a group of mothers diagnosed with HIV and the mothers reported reduced feelings of depression and stress ${ }^{25}$. Infant massage also supports fathers in their new role as a parent as fathers who took part in an infant massage programme reported lower levels of stress ${ }^{27}$.

Various high-quality randomised controlled trials have been conducted highlighting the above-mentioned benefits of infant massage for infants and parents ${ }^{28-30}$. The effect of infant massage on the developmental milestones of preterm infants is, however, not clear and this study was designed to investigate this aspect. Three objectives were addressed: firstly, to determine the developmental outcomes for two groups of premature infants, those who received infant massage and those who did not; secondly, to describe barriers and facilitators identified by parents on the implementation of a hospital- and homeadministered infant massage programme into their daily routines; and finally, to determine the association between compliance of parentadministered infant massage and the developmental outcomes of premature infants at four to six months actual age.

\section{METHOD}

\section{Study design}

This study made use of a quantitative, blinded, randomised control trial design. Figure I (below) shows the RCT flow chart of the recruitment, randomisation, assessment and re-assessment of the experimental and control groups.

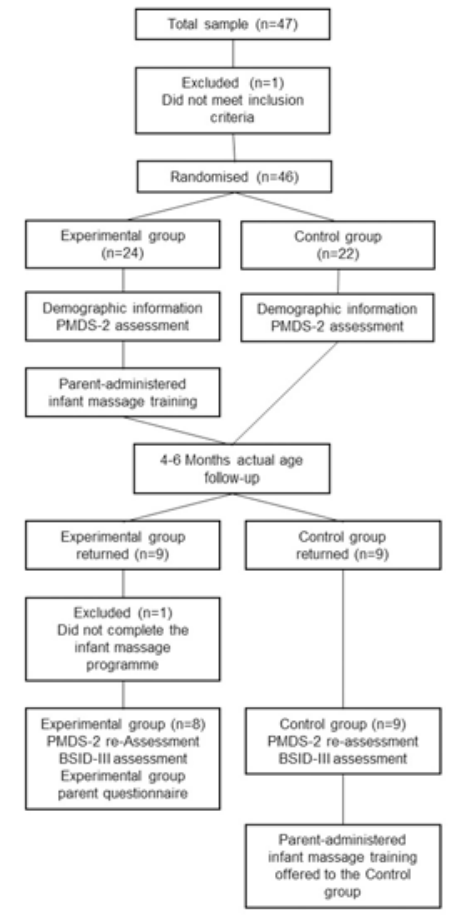

Figure I: Flow chart for the randomised controlled trial on parent-administered infant massage

\section{Population and sampling procedures}

The study population consisted of infants who were born prematurely at Chris Hani Baragwanath Academic Hospital, Gauteng and their parents. This study made use of purposive sampling. The inclusion criteria included infants born between 28- and 36-weeks gestational age and aged between 32 - and 36 -weeks gestational age 
at the time of recruitment. Infant massage is not recommended for infants younger than 32 weeks gestation or for infants weighing less than $1000 g^{31}$. Infants who had neurological diagnoses or genetic complications were excluded from the study as well as infants whose parents were younger than 18 years old or who were receiving kangaroo care. Parents had to be proficient in speaking, reading and writing English. Infants and parents, who met the inclusion criteria and were in the wards at the time of screening, were invited to participate in the study.

The sample size was calculated at 43 participants per group based on an effect size of $10 \%$, a standard deviation of 15 on the BSID-III assessment with a power of $80 \%{ }^{32}$. Participants were recruited over a six-month period and the final sample size was 46 participants. Parents who agreed to participate in the study were assigned to either the experimental or the control group using simple randomisation with a computer-generated randomiser. The researcher was blinded to the group participants had been assigned to, to ensure all assessments of the infants were unbiased. The researcher conducted all assessments while a research assistant, who is an internationally certified infant massage instructor, trained the parents in the experimental group. The infant massage protocol consisted of a daily, I0-15 minutes session administered by trained parents. There is no current best practice standard for infant massage ${ }^{19}$. The dosage for this study was based on the protocol of similar studies using one or two I5-minute sessions per day ${ }^{16,33}$. These short sessions are manageable for parents to fit into their daily routine. The infant's lower limbs, stomach, chest and upper limbs were massaged. Even though the IAIM prescribes the use of a cold pressed vegetable oil in the practice of infant massage ${ }^{22}$, insufficient evidence is available on the effect of different lubricants on the skin of an infant ${ }^{19}$. The use of oil on the skin of an infant may be associated with an adverse effect on the barrier function of the $\operatorname{skin}^{19}$ and infants may even develop an allergic rash during the massage practice ${ }^{34}$. No oil was therefore used in this study. Parents used the same baby cream or lotion they used as part of the infant's bath routine.

\section{Data collection}

Data were collected at two points in time: on recruitment and post discharge at four- and six-months actual age when the infants returned for their first follow-up appointment with the doctors. At the initial assessment, demographic information was collected by the researcher using a questionnaire developed by the researcher that was divided into parent demographics and infant demographics. The questionnaire included information about the infant's gestational age, weight, head circumference, length, corrected age and any neonatal complications. Information about the mother included her age, any complications she may have had while pregnant, highest level of education and whether she had returned to work. Medical records were also reviewed by the researcher and relevant information collected regarding birth and medical history.

All infants were assessed by the researcher using the Peabody Developmental Motor Scales - Second Edition (PDMS-2). The assessment provided a baseline functional evaluation of the infant and the same test was completed with the infant at four to six months actual age. The PDMS- 2 is a test that can be used from birth to five years of age and has been shown to be reliable and valid in assessing the motor capabilities of infants and children. The PDMS-2 was shown to have an excellent internal consistency of 0.92 (Cronbach's alpha coefficient) and an excellent test-retest reliability of $0.98^{35}$. The test consists of six subtests, namely: Reflexes, Locomotion, Stationary, Grasp, Object Manipulation and Visual-Motor Integration. The PDMS-2 scores were converted to standard scores which allowed for the comparison of scores of infants with different ages.

At the follow-up assessment, the Bayley Scales of Infant and Toddler Development - Third Edition (BSID-III) was also used to assess the infants, as this is reported to be the gold standard of infant and toddler developmental assessments ${ }^{36}$. The BSID-III scores an infant's development on five scales, namely: Cognitive, Language, Motor, Social-Emotional and Adaptive Behavior ${ }^{36}$. Although the BSID-III was developed and referenced in the United States of America, a 2013 study proved that the test was valid for use with South African urban children ${ }^{37}$.

The parents of the infants assigned to the experimental group were required to complete an additional questionnaire, in English, on the barriers and facilitators related to executing the massage programme. This was administered by the research assistant after the child had been assessed by the researcher.

\section{Data analysis}

Demographic data were analysed using descriptive and inferential statistics to ensure the two groups of infants were comparable. Data collected from the developmental screening tools were summarized using means and standard deviations of the standard scores of the test. Standard scores were used to analyse the data as these yielded similar results when determining the concurrent validity of the BSID-III and the PDMS-2 ${ }^{38}$. Due to the small sample size the data were not normally distributed and comparisons between the two groups were made using the unpaired t-test. Cohen's d-test was used to determine the clinical effect size between the two groups on the standardised scores of the PDMS-2 and the BSID-III. Linear regressions were used to compare the effect that frequency of massage had on test scores.

\section{Ethical clearance and informed consent}

Ethical clearance was obtained from the University of the Witwatersrand's Human Research Ethics Committee prior to participant recruitment and data collection with clearance number MI604I8. Parents, who were willing to participate in the study, were given an information letter explaining the study and signed informed consent prior to participation. All participants were given a participant code so that their personal information was kept separately from their assessment result data to ensure confidentiality. Consent was obtained from the hospital's medical advisory committee as well as the Heads of Department of Paediatrics and Occupational Therapy.

\section{RESULTS}

The study sample consisted of 46 infants and their parents who completed the initial data collection. Due to a high attrition rate, only 17 infants were reassessed at the follow-up appointment. Therefore, results reported are for 17 infants and their parents that completed the research process, unless otherwise stated. There were nine participants in the control group and eight participants in the experimental group.

\section{Demographics}

The control and experimental groups of infants were comparable in terms of their demographics and anthropometrics as there 
Table I: Follow-up infant demographics $(n=17)$

\begin{tabular}{|c|c|c|c|c|}
\hline \multirow[t]{2}{*}{ Variable } & \multicolumn{3}{|c|}{ Group } & \multirow[t]{3}{*}{ p-Value } \\
\hline & $\begin{array}{l}\text { Total } \\
(n=17)\end{array}$ & $\begin{array}{c}\text { Control } \\
(\mathrm{n}=9)\end{array}$ & $\begin{array}{l}\text { Experi- } \\
\text { mentiol }\end{array}$ & \\
\hline & \multicolumn{3}{|c|}{ n (\%) } & \\
\hline $\begin{array}{l}\text { Gender } \\
\text { Menale } \\
\text { Male }\end{array}$ & फி (59\%) & $5(56 \%)$ & $5(63 \%)$ & 1.000 \\
\hline $\begin{array}{l}\text { Mode of deliyery } \\
\text { Catura vaginal } \\
\text { Caesarean section }\end{array}$ & $\begin{array}{l}6(35 \%) \\
11\end{array}$ & $\begin{array}{l}4(44 \%) \\
5\end{array}$ & 2 (35\%) & 0.620 \\
\hline $\begin{array}{l}\text { Neonatal } \\
\text { complications } \\
\text { NAN } \\
\text { NN } \\
\text { NDS } \\
\text { Other } \\
\text { None }\end{array}$ & $\begin{array}{l}2(12 \%) \\
0(6 \% \%) \\
136 \% \%) \\
0(18 \%) \\
3(18 \%)\end{array}$ & $\begin{array}{l}1(11 \%) \\
0(0 \% \%) \\
8(11 \%) \\
8(8 \%) \\
8(0 \%)\end{array}$ & $\begin{array}{l}1(13 \%) \\
8(8 \% \%) \\
5(63 \%) \\
0(38 \%)\end{array}$ & 0.087 \\
\hline
\end{tabular}

$p \leq 0.05$ statistical significance*

Neonatal jaundice (NNJ)

Transient tachypnoea of the new-born (TTN)

Necrotising enterocolitis (NEC)

Respiratory distress syndrome (RDS)

were no statistically significant differences between the two groups at the time of their follow-up assessment (Table I and Table II). All the parents who returned for follow-up appointments were the mothers of the infants (I00\%; $n=17)$ and there was no statistically significant difference between the mothers of the two groups in terms of age $(p=0.490)$, highest level of education $(p=0.289)$, employment status $(p=0.620)$ or maternal conditions $(p=0.637)$.

\section{Peabody Developmental Motor Scales - Second Edition test results}

The initial test scores of the PDMS- 2 were comparable between the control and experimental groups of infants indicating that they were developmentally functioning on a similar level at the start of the study. Follow-up test scores indicated the experimental group performed better in the Reflexes, Stationary, Grasping and VisualMotor Integration subtests while the control group performed better in the Locomotion subtest. Large clinical effect sizes were noted in the Reflexes $(\mathrm{d}=0.78)$ and the Visual-Motor Integration $(\mathrm{d}=\mathrm{l} .09)$ subtests. When comparing initial standardised test scores to the follow-up test scores to determine improvement in milestone attainment, the experimental group performed better in all subtests other than Grasping, and large clinical effect sizes were noted in the Reflexes $(\mathrm{d}=0.73)$ and Visual-Motor Integration $(\mathrm{d}=\mathrm{I} .03)$ subtests (Table III. p40).

\section{Bayley Scales of Infant and Toddler Development - Third Edition test results}

The BSID-III test scores indicated the experimental group per-
Table II: Follow-up infant anthropometrics $(n=17)$

\begin{tabular}{|c|c|c|c|c|}
\hline \multirow[t]{3}{*}{ Variable } & \multicolumn{3}{|c|}{ Group } & \multirow{3}{*}{$\begin{array}{c}\text { p- } \\
\text { Value }\end{array}$} \\
\hline & $\begin{array}{c}\text { Total } \\
(n=17)\end{array}$ & $\begin{array}{l}\text { Control } \\
(n=9)\end{array}$ & $\begin{array}{l}\text { Experimental } \\
\quad(n=8)\end{array}$ & \\
\hline & $\begin{array}{l}\text { Mean } \\
\text { (SD) }\end{array}$ & Mean (SD) & Mean (SD) & \\
\hline $\begin{array}{l}\text { Gestational } \\
\text { age (weeks) }\end{array}$ & $\begin{array}{l}33.06 \\
(1.7 I) \\
\end{array}$ & $33.33(1.22)$ & 32.75 (2.19) & 0.589 \\
\hline $\begin{array}{l}\text { Birth weight } \\
\text { (g) }\end{array}$ & $\begin{array}{l}1681.18 \\
(288.34)\end{array}$ & $\begin{array}{l}1636.67 \\
(212.31)\end{array}$ & $\begin{array}{l}\mid 731.25 \\
(364.85)\end{array}$ & 0.736 \\
\hline $\begin{array}{l}\text { Actual age } \\
\text { (months) }\end{array}$ & $5.50(0.87)$ & $5.46(1.06)$ & $5.54(0.67)$ & 0.961 \\
\hline $\begin{array}{l}\text { Corrected } \\
\text { age (months) }\end{array}$ & $3.95(1.01)$ & $3.97(1.18)$ & $3.94(0.86)$ & 1.000 \\
\hline $\begin{array}{l}\text { Weight at } \\
\text { follow-up (g) }\end{array}$ & $\begin{array}{c}5811.76 \\
(1229.61)\end{array}$ & $\begin{array}{c}5791.11 \\
(1206.35)\end{array}$ & $\begin{array}{c}5835.00 \\
(1338.47)\end{array}$ & 0.923 \\
\hline $\begin{array}{l}\text { Length at } \\
\text { follow-up } \\
(\mathrm{cm})\end{array}$ & $\begin{array}{l}54.88 \\
(4.50)\end{array}$ & $58.25(3.77)$ & $58.38(5.40)$ & 0.874 \\
\hline $\begin{array}{l}\text { Head circum- } \\
\text { ference at } \\
\text { follow-up (cm) }\end{array}$ & $\begin{array}{l}38.88 \\
(2.02)\end{array}$ & $40.38(2.13)$ & $42.25(1.49)$ & 0.063 \\
\hline
\end{tabular}

$p \leq 0.05$ statistical significance*

formed better in the Cognition, Language and Motor subtests (Table IV. p40). The control group performed better in the SocialEmotional and the Adaptive Behavior subtests. A medium clinical effect size was noted on the Cognition $(d=0.58)$ subtest. The Social-Emotional and Adaptive Behavior subtests showed negative, small and medium clinical effect sizes ( -0.22 and -0.56 respectively) indicating that infants in the control group performed better than the experimental group infants.

\section{Experimental group parent questionnaire results}

The answers parents in the experimental group provided on the parent questionnaire indicated they carried out the massage anywhere between two and seven times per week. The mothers identified barriers to carrying out the massage as: looking after other children, busy with other household tasks, the baby was asleep, and the baby was very small - I was scared to do the massage. All the mothers reported they found the massage enjoyable; the most common reasons for enjoyment were the one-on-one time they had with their infant and their infant's reaction to the massage. The majority of mothers $(75 \%, n=6)$ reported the massage sessions lasted between 16 and 30 minutes. Only three of the mothers $(38 \%, n=3)$ reported the massage sessions were too long and they found it difficult to fit the massage into their daily routines. The majority of the mothers $(75 \%, n=6)$ carried out the massage either before or after the infant's bath time. All of the mothers found the massage satisfying. When asked to elaborate on their satisfaction, mothers reported the massage was a bonding experience, it was beneficial for the infant and it was relaxing for themselves. 
Table III: Comparison of change in Standard Scores on the Peabody Developmental Motor Scale-Second Edition subtests from initial to follow-up scores $(n=17)$

\begin{tabular}{|l|c|c|c|c|c|}
\hline \multirow{2}{*}{ Subtest } & \multicolumn{3}{|c|}{ Group } & \multirow{2}{*}{ p-Value } & \multirow{2}{*}{ Cohen's d } \\
\cline { 2 - 5 } & $\begin{array}{c}\text { Total } \\
(\mathbf{n = 1 7 )}\end{array}$ & $\begin{array}{c}\text { Control } \\
(\mathbf{n = 9 )}\end{array}$ & $\begin{array}{c}\text { Experimental } \\
(\mathbf{n = 8})\end{array}$ & \\
\hline Reflexes & $1.59(1.01)$ & $1.22(1.30)$ & $2,00(0.76)$ & 0.240 & $0.73^{\circ 00}$ \\
\hline Stationary & $2.76(1.06)$ & $2.45(0.53)$ & $3.13(1.46)$ & 0.212 & $0.62^{\circ \circ}$ \\
\hline Locomotion & $2.53(0.92)$ & $2,45(1.01)$ & $2.64(0.92)$ & 0.758 & $0.19^{\circ}$ \\
\hline Grasping & $-1.00(1.64)$ & $-0.89(1.97)$ & $-1.13(1.46)$ & 1.000 & $-0.14^{\circ}$ \\
\hline $\begin{array}{l}\text { Visual-motor } \\
\text { integration }\end{array}$ & $4.53(0.92)$ & $4.11(0.60)$ & $5.00(1.07)$ & 0.054 & $1.03^{\circ 00}$ \\
\hline
\end{tabular}

$\mathrm{p} \leq \mathbf{0 . 0 5}$ statistical significance* $d \geq 0.8$ large clinical effect size soo $^{000}$ $\mathrm{d} \geq \mathbf{0 . 5}$ medium clinical effect size ${ }^{\circ 0}$ $d \geq 0.2$ small clinical effect size ${ }^{\circ}$
Table IV: Follow-up Standard Scores for the Bayley Scales of Infant and Toddler DevelopmentThird Edition $(n=17)$

\begin{tabular}{|l|c|c|c|c|c|}
\hline & \multicolumn{3}{|c|}{ Group } & \multirow{2}{*}{ p-Value } & \multirow{2}{*}{ Cohen's d } \\
\cline { 1 - 4 } & $\begin{array}{c}\text { Total partici- } \\
\text { Vants (n= 17) }\end{array}$ & Conffel \&)oup & $\begin{array}{c}\text { Experimental } \\
\text { group (n=8) }\end{array}$ & \\
\hline & \multicolumn{3}{|c|}{ Mean (SD) } & \\
\hline Cognition & $9.29(2.37)$ & $8.67(2.16)$ & $10.00(2.40)$ & 0.326 & $0.58^{\circ \circ}$ \\
\hline Language & $17.59(2.61)$ & $17.56(2.87)$ & $17.63(2.29)$ & 0.845 & 0.03 \\
\hline Motor & $19.53(3.97)$ & $18.78(3.94)$ & $20.38(3.84)$ & 0.528 & $0.41^{\circ}$ \\
\hline $\begin{array}{l}\text { Social- } \\
\text { emotional }\end{array}$ & $16.29(2.61)$ & $16.56(2.91)$ & $16.00(2.18)$ & 0.487 & $-0.22^{\circ}$ \\
\hline $\begin{array}{l}\text { Adaptive } \\
\text { behavior }\end{array}$ & $69.76(7.03)$ & $71.56(7.15)$ & $67.75(6.23)$ & 0.228 & $-0.56^{\circ 0}$ \\
\hline
\end{tabular}

\author{
$p \leq 0.05$ statistical \\ significance * \\ $\mathrm{d} \geq 0.8$ large clinical \\ effect size ${ }^{000}$ \\ $\mathrm{d} \geq \mathbf{0 . 5}$ medium clinical \\ effect size ${ }^{\circ 0}$ \\ d $\geq 0.2$ small clinical \\ effect size ${ }^{\circ}$
}

\section{Correlation between frequency of massage and developmental test scores}

\section{Peabody Developmental Motor Scales - Second Edition}

There was a high positive correlation between the frequency of massage sessions and the Reflexes $(r=0.6 \mathrm{l})$ subtest on the PDMS-2. There were two negative correlations on the Locomotion $(r=-0.1 \mathrm{I})$ and Grasping ( $r=-0.07)$ subtests; these were, however, very low and not statistically significant.

\section{Bayley Scales of Infant and Toddler Development - Third Edition}

There were moderate negative to very low correlations on the Language $(r=-0.4 \mathrm{I})$, Motor $(r=-0.29)$ and Adaptive Behavior $(r=-$ $0.10)$ subtests (Table $V$ ). There were positive correlations on the Cognition $(r=0.33)$ and Social Emotional $(r=0.75)$ subtests (Table $\mathrm{V})$. The high positive correlation on the Social Emotional subtest was found to be statistically significant $(p=0.033)$ (Table V. p4I).

\section{DISCUSSION AND IMPLICATIONS}

The infants from both experimental and control groups were comparable in terms of their demographic and anthropometric factors. There was also no statistically significant difference between the two groups' initial PDMS-2 assessment results, indicating infants from both groups were developmentally on par prior to the intervention. Differences in the developmental outcomes between the two groups, at the time of the follow-up appointment, could therefore not be attributed to demographic or anthropometric factors or initial developmental levels.

When comparing the change in results of the control and experimental groups from the initial and follow-up assessments, large and medium clinical effect sizes were identified in the Reflexes, Stationary and Visual-Motor Integration subtests of the PDMS-2 with the experimental group performing better on these subtests. Reflexes are the foundation for later voluntary movement and motor skills ${ }^{39}$, which infants need to learn as they grow, assume different roles and take part in different occupations throughout their life. The occupations expected of the new-born include social play, feeding, exploration and procuring which all require motor skills'. Motor skills have further been shown to have an impact on skills such as mathematical and reading abilities when children start school ${ }^{7}$. The fact that preterm infants, who received parent-administered massage in this study, performed better in the Reflexes subtest is an important finding as it provides the basis for not only later motor development, but also school-based skills.

The Stationary subtest of the PDMS-2 looks at an infant's head and trunk control abilities. It has been suggested that premature infants have decreased abilities when it comes to head control developmental milestones ${ }^{40}$. Showing a medium clinical effect in this area, following the implementation of the massage programme, could have further positive implications for other stationary, gross motor milestones of premature infants such as sitting and standing. These two positions are especially important as most daily occupations of children and adults take place in sitting and standing. Furthermore, the ability to control ones' trunk and maintain upright positions, such as sitting, has been shown to be strongly correlated to the reaching ability of an 
Table V: Linear regression between frequency of massage and BSID-III Standard Scores

\begin{tabular}{|c|c|c|c|c|c|c|}
\hline & Cognition & Language & Motor & Social-emotional & $\begin{array}{l}\text { Adaptive } \\
\text { behavior }\end{array}$ & $p \leq 0.05$ statistical significance* \\
\hline $\begin{array}{l}\text { Correlation } \\
\text { coefficient }(r)\end{array}$ & 0.33 & $-0.4 I$ & -0.29 & 0.75 & -0.10 & $\begin{array}{l}r=0.2-0.4 \text { low correlation } \\
r=0.4-0.6 \text { moderate correlation }\end{array}$ \\
\hline $\mathrm{p}$ - value & 0.432 & 0.307 & 0.488 & $0.033 *$ & 0.806 & $\begin{array}{l}r=0.6-0.8 \text { high correlation } \\
r=0.8-1.0 \text { very high corrrel }\end{array}$ \\
\hline
\end{tabular}

infant ${ }^{41}$. If infants could assume and maintain these positions sooner, they would be able to learn new skills in these positions such as hand function skills which would in turn affect their ability to participate in all occupations.

Visual-motor integration, is required for two critical skills that children will use throughout their schooling careers and into their adult lives namely: handwriting and mathematics. These skills have been shown to be affected in premature infants ${ }^{42}$. It was further concluded in a 2018 study that children who were born prematurely are at risk of dysfunction in many higher-order visual tasks ${ }^{9}$. Children who present with visual-motor integration disorders are often only identified at crèche or school age when more structured visual-motor tasks are required of them. The finding in this study of a large positive clinical effect size related to visual-motor integration, in infants who have received infant massage, is important as early intervention could assist with minimising the problems premature infants often have in these areas later on.

There were no statistically significant differences between the results of the BSID-III of the two groups of infants. Medium clinical effect sizes, in favour of the experimental group, were however identified for the Cognition subtest. In a 2014 study, it was found that up to two years of age, premature infants score statistically lower than their full-term counterparts when tested on the BSID-III Cognition subtest ${ }^{43}$. The same study showed that infants do tend to improve from the initial cognitive impact of prematurity, however, the effect of the initial cognitive disadvantage over a longer period was not studied ${ }^{43}$. Given the intricacies of development, one could argue that cognition plays an important role in overall development, therefore, a medium clinical effect from infant massage in these at-risk premature infants is a potentially important finding.

The small to medium clinical effect size on the Motor subtest $(0.4 I)$ of the BSID-III, indicate the experimental group had performed better on this subtest. The Motor subtest is divided into gross motor and fine motor components. Gross motor items include head control in different positions, rolling abilities, prone play and sitting abilities while fine motor items include tracking abilities, bringing hands to mouth and grasping. As discussed above these fine and gross motor milestones impact on development and participation in occupations such as dressing, hygiene, play activities and school tasks. Premature infants further have different tracking abilities and a delay in their development of smooth pursuit eye movements when compared to their full-term counterparts ${ }^{44}$. Improved visual abilities could positively affect visual-motor integration skills as discussed above. A positive, even if small, clinical effect in the motor area is therefore important to note.

The control group performed better on the Social-emotional subtest (small negative clinical effect size) and the Adaptive Behavior subtest (medium negative effect size). These two subtests on the BSID-III are self-reported parent questions and the results should therefore be analysed with caution. Further investigations are required to establish a possible reason for these findings.
The execution of home programmes is most successful when they can be integrated into a caregiver and infant's daily routine ${ }^{45}$. As shown in this study, the majority of mothers reported that the daily massage sessions were not too long to fit into their daily routines and three quarters of mothers were found to have integrated the massage sessions into their infant's bath time. The ability to integrate the massage programme into their daily routine would be a facilitator to making the programme successful as described in a 2014 study ${ }^{45}$. When asked about their satisfaction with the massage programme, all the mothers were either satisfied or very satisfied and they indicated they enjoyed the massage. A 2012 study on the link between treatment satisfaction and adherence, compliance and persistence suggested that the greater satisfaction there is with a programme, the greater the compliance to that programme is ${ }^{46}$.

The mothers in this study noted a few barriers to completing daily massage sessions with their infant. A quarter of the mothers reported they were looking after other children and could therefore not complete the massage sessions daily. Looking after other children and parental stress levels can affect the compliance to a home exercise programme ${ }^{47}$. This was evident in this study as one mother had a tragic event in their family and therefore did not complete the massage programme at home. Another mother also noted she was scared to do the massage as the baby was very small, even though all mothers had extensive training on infant massage by the research assistant. This is in line with the anxiety often experienced by parents of premature infants ${ }^{48}$. Infant massage has, however, been shown to decrease parental stress ${ }^{25}$. Parent-administered infant massage could therefore be beneficial for anxious mothers to assist with their anxiety levels and their new role of, not only mother, but mother of a premature infant.

When the frequency of massage sessions was correlated with the Social-emotional subtest (on the BSID-III), a statistically significant positive correlation was found. Infant-parent bonding and attachment affect the infant's life-long social development ${ }^{49}$. The parent is described as the first bond infants will have and this is the basis for their future emotional development ${ }^{49}$. Parents were taught, during instruction on infant massage, to read their infants' cues and respond appropriately. Parents were taught to look for signs that their infants are not coping with the massage such as crying, avoiding eye-contact, back arching or hand splaying and to respect the infants' reactions and stop the massage sessions. Alternatively, when the infant is looking at them, holding their own hands, cooing or smiling, it is a sign that the infant was enjoying the massage and parents could continue with the session. It would therefore make sense that there was a positive correlation between frequency of massage and social-emotional development as the parents are responding to the infants' earliest form of communication which is one of the main occupations of an infant. This would further be impacted by the fact that infant massage is taught to parents as a one-on-one bonding time with their child, free from distractions. Therefore, the higher the frequency of the massage sessions, the more time the parent was giving their infant their undivided attention. On the correlation of the results 
with frequency of massage, it was further noted that the Cognition subtest had a low positive correlation while the Language subtest had a moderate negative correlation. Language and cognition are interlinked ${ }^{50}$ and further investigation would be required into this finding.

A limitation to this study was that the small sample size lowered the chances of finding statistical significance in results between the experimental and control groups. Many of the mothers who did not return for follow-up could not be reached telephonically as their phones were often off or had been disconnected. This indicates that there were possibly socio-economic concerns for these mothers which should be considered in future studies. A further limitation was that the infants were only studied over their first four to six months of life, whereas a longitudinal study would have produced more data to study the long-term effects of infant massage. The final limitation was that the parent questionnaire was mainly quantitative and did not give mothers an opportunity to add their own thoughts and feelings.

\section{CONCLUSION}

Positive touch, as one component of neurodevelopmental supportive care, has various benefits for premature infants and their parents. Parent-administered infant massage is one form of positive touch. The results of this study confirm a positive clinical effect on the developmental milestones of premature infants, who received parent-administered infant massage, when assessed at four to six months actual age in comparison with infants who had did not receive the intervention. Facilitators to parent-administered infant massage, that supported the mothers in carrying out the massage sessions, have been identified and include aspects such as the ease of integrating the programme into daily routines and enjoyment of the programme. Barriers to completing the massage sessions included parental stress, performing other household chores and looking after other children. Mothers were in general positive about the massage sessions with their infants. A strong positive correlation was established between the frequency of massage sessions and the social-emotional development of premature infants. This links to parent-infant bonding with mothers being able to read the infant's cues during the massage process and respond appropriately. The findings of the study are in favour of parentadministered infant massage for premature infants to assist with the attainment of their developmental milestones as well as promoting the parent-infant bonding.

\section{ROLE OF AUTHORS}

Megan Campbell took the lead in writing the manuscript. Lizelle Jacobs provided critical feedback and helped shape the research, analysis, and manuscript. Both authors contributed to the final version of the manuscript. Lizelle Jacobs supervised this research project.

\section{REFERENCES}

I. Vergara E, Bigsby R. Developmental and Therapeutic Interventions in the NICU. Baltimore: Brookes; 2004.

2. Vinall J, Grunau R. Impact of repeated procedural pain-related stress in infants born very preterm. Padiatric Research. 2014;75(5):584-7. doi: https://doi.org/10.1038/pr.2014.16.

3. Formiga CKMR, Linhares MBM. Motor development curve from 0 to 12 months in infants born preterm. Acta Paediatrica. 201 I; 100(3):37984. doi: https://doi.org/10.1 I I I/j.165I-2227.2010.02002.x.

4. Ballot DE, Ramdin T, Rakotsoane D, Agaba F, Chirwa T, Davies VA, et al. Assessment of developmental outcome in very low birth weight infants in southern Africa using the Bayley scales of infant development (III). BMJ paediatrics open. 2017; I(I):I-7. doi: https://doi.org/10.1 136/bmjpo-2017-000091.

5. Guerra CC, de Moraes Barros MC, Goulart AL, Fernandes LV, Kopelman $\mathrm{Bl}$, dos Santos AM. Premature infants with birth weights of I500-1999 g exhibit considerable delays in several developmental areas. Acta Paediatrica. 20 I4; I03(I):el-e6. doi: https://doi.org/I0.I I I I/apa. 2430.

6. Pinheiro R, Martinez C, Fontaine A. Visual motor integration and overall development of preterm and at term children at the beginning of schooling. Journal of Human Growth and Development. 2014;24(2): 18I-7. doi: https://doi.org//0.7322/jhgd.8I 037.

7. Cameron C, Cottone E, Murrah W, Grissmer D. How are motor skills linked to children's school performance and academic achievement? Child Development Perspectives. 2016;10(2):93-8. doi: https://doi.org/10.1 I I I/cdep. 12168.

8. Bolk J, Kaul Y, Hellstrom-Westas L, Stjernqvist K, Padilla N, Serenius F, et al. National population-based cohort study found that visualmotor integration was commonly affected in extremely preterm born children at six-and-a-half years. Acta Paediatricia. 20 I 8; 107(5):83I-7. doi: https://doi.org/10.1 I I I/apa.1423I.

9. Leung MP, Thompson B, Black J, Dai S, Alsweiler JM. The effects of preterm birth on visual development. Clinical and Experimental Optometry. 20 I8; I0I(I):4-I2. doi: https://doi.org/I0. I I I I/cxo. I 2578.

10. Case-Smith J, O'Brien JC. Occupational therapy for children-E-Book: Elsevier Health Sciences; 2013.

II. Legendre V, Burtner P, Martinez K, Crowe T. The evolving practice of developmental care in the neonatal unit: A systematic review. Physical \& Occupational Therapy in Pediatrics. 201 1;31(3):315-38. doi: https://doi.org/10.3109/01942638.201 I.556697.

12. Westrup B. Newborn Individualized Developmental Care and Assessment Program (NIDCAP) - Family-centered developmentally supportive care. Early Human Development. 2007;83(7):443-9. doi: https://doi.org/10.1016/j.earlhumdev.2007.03.006.

13. Altimier LB. Neuroprotective core measure I: the healing NICU environment. Newborn and Infant Nursing Reviews. 20 I5; I5(3):91-6. doi: https://doi.org/10.1053/j.nainr.2015.06.014.

14. Bader L. Brain-oriented care in the NICU: A case study. Neonatal Network. 2014;33(5):263-7. doi: https://doi.org//0.1891/0730-0832.33.5.263 .

15. Lubbe W, Van der Walt C, Klopper H. Integrative literature review defining evidence-based neurodevelopmental supportive care of the preterm infant. The Journal of Perinatal \& Neonatal Nursing. 2012;26(3):25I-9. doi: https://doi.org/10.1097/JPN.0b0 I 3e3182650b7e.

16. Field T, Diego M, Hernandez-Reif M. Preterm infant massage therapy research: A review. Infant Behaviour and Development. 2010;33(2): I I5-24. doi: https://doi.org/10.1016/j.infbeh.2009.12.004.

17. Diego M, Field T, Hernandez-Reif M. Preterm infant weight gain is increased by massage therapy and excercise via different underlying mechanisms. Early Human Development. 2014;90(3): 137-40. doi: https://doi.org/10.1016/j.earlhumdev.2014.01.009.

18. Faure M, Richardson A. Baby Sense. Welgemoed: Metz Press; 2014.

19. Cooke A. Infant massage: The practice and evidence-base to support it. British Journal of Midwifery. 2015;23(3): I66-70.

20. Lubbe W. Prematurity Adjusting your Dream. Pretoria: Little Steps; 2008.

21. Duhn $L$. The importance of touch in the development of attachment. Advances in Neonatal Care. 2010;10(6):294-300. doi: https://doi.org/10.1097/ANC.0b013e3 I8Ifd2263.

22. McClure V. Infant Massage A Handbook for Loving Parents. London: Souvenir Press Ltd; 2008. 
23. Bader L. The Ladder Approach. Nebraska: Training manual from author; 2012.

24. Diego MA, Field T, Hernandez-Reif M, Deeds O, Ascencio A, Begert $G$. Preterm infant massage elicits consistent increases in vagal activity and gastric motility that are associated with greater weight gain. Acta Paediatrica. 2007;96(II):I588-9I.

doi: https://doi.org//0.I I I I/j. I65I-2227.2007.00476.x.

25. Oswalt K, Biasini F. Effects of infant massage on HIV-infected mothers and their infants. Journal for Specialists in Pediatric Nursing. 2011;16(3):169-78. doi:

https://doi.org/10.1 I I I/j. I744-6155.201 I.00291.x.

26. Vincente $S$, Verissimo $M$, Diniz E. Infant massage improves attitudes toward childbearing, maternal satisfaction and pleasure in parenting. Infant Behavior and Development. 2017;49:1 I4-9. doi: https://doi.org/10.1016/j.infbeh.2017.08.006.

27. Cheng C, Volk A, Marini Z. Supporting fathering through infant massage. The Journal of Perinatal Education. 201 I;20(4):200-9. doi: https://doi.org//0.1891/1058-1243.20.4.200.

28. Diego MA, Field T, Hernandez-Reif M. Vagal activity, gastric motility, and weight gain in massaged preterm neonates. The Journal of pediatrics. 2005; I47(I):50-5. doi: https://doi.org/10.1016/j.jpeds.2005.02.023.

29. Field T, Diego M. Vagal activity, early growth and emotional development. Infant Behavior and Development. 2008;3I(3):36I-73. doi: https://doi.org/10.1016/j.infbeh.2007.12.008.

30. Ho YB, Lee RS, Chow CB, Pang MY. Impact of massage therapy on motor outcomes in very low-birthweight infants: Randomized controlled pilot study. Pediatrics International. 2010;52(3):378-85. doi: https://doi.org/I0. I I I I/j. I442-200X.2009.02964.x.

3I. Chan A, So W, Shih F, Chui K. Development of an evidence-based guideline for preterm infant massage by parents. International Journal of Complementary Alternative Medicine. 2015;2(I):00042. doi: https://doi.org/10.15406/ijcam.2015.02.00042.

32. Greene MM, Patra K, Silvestri JM, Nelson MN. Re-evaluating preterm infants with the Bayley-III: patterns and predictors of change. Research in developmental disabilities. 2013;34(7):2107-17. doi: https://doi.org/10.1016/j.ridd.2013.04.00I.

33. Field T. Massage therapy research review. Complementary therapies in clinical practice. 2014;20(4):224-9. doi: https://doi.org/10.1016/j. ctcp.2014.07.002

34. Kulkarni A, Kaushik J, Gupta P, Sharma H, Agrawal R. Massage and touch therapy in neonates: The current evidence. Indian Pediatrics. 20 10;47(9):77I-6. doi: https://doi.org/10.1007/s I33 I2-010-0I I4-2.

35. Tavasoli A, Azimi P, Montazari A. Reliability and validity of the Peabody Developmental Motor Scales-second edition for assessing motor development of low birth weight preterm infants. Pediatric Neurology. 20I4;5I (4):522-6.

doi: https://doi.org/10.1016/j.pediatrneurol.2014.06.010.

36. Yildirum Z, Aydinli N, Ekici B, Tatli B, Caliskan M. Can Alberta infant motor scale and milani comparetti motor development screening test be rapid alternatives to bayley scales of infant development-Il at highrisk infants. Annals of Indian Academy of Neurology. 20 I2; 15(3): 196 9. doi: https://doi.org/I0.4I03/0972-2327.997/4.

37. Radermeyer $\mathrm{V}$, Jacklin L. A study to evaluate the performance of black South African urban infants on the Bayley Scales of Infant Development III. South African Journal of Child Health. 2013;7(2):54-9. doi: https://doi.org//0.7/96/sajch.547.

38. Connolly BH, McClune NO, Gatlin R. Concurrent validity of the Bayley-III and the Peabody developmental motor scale-2. Pediatric Physical Therapy. 2012;24(4):345-52.

doi: https://doi.org/10.1097/PEP.0b0 I 3e318267c5cf.
39. Salehi S, Sheikh M, Talebrokni F. Comparison exam of Gallahue's Hourglass Model and Clark and Metcalfe's the Mountain of Motor Development Metaphor. Advances in Physical Education. 2017;7(03):21 7-33. doi: https://doi.org/10.4236/ape.2017.73018.

40. Babik I, Galloway J, Lobo M. Infants born preterm demonstrate impaired exploration of their bodies and surfaces throughout the first 2 years of life. Physical Therapy. 2017;97(9):915-25. doi: https://doi.org/10.1093/ptj/pzx064.

41. Rachwani J, Santamaria V, Saavedra S, Woollacott, MH. The development of trunk control and its relation to reaching in infancy: A longitudinal study. Frontiers in Human Neuroscience. 20I5;9(94): I- 12. doi: https://doi.org/10.3389/fnhum.2015.00094.

42. Aarnoudse-Moens C, Weisglas-Kuperus N, van Goudoever J, Oosterlaan J. Meta-analysis of neurobehavioral outcomes in very preterm and/or very low birth weight children. Pediatrics. 2009; 124(2):717-29. doi: https://doi.org/10.1542/peds.2008-2816.

43. Ionio CR, E, Confalonieri E, Dallatomasina C, Mascheroni, E, Bonanomi A, Sora N, Falautano M, et al. Paths of cognitive and language development in healthy preterm infants. Infant Behaviour and Development. 2014;44:199-207. doi: https://doi.org/10.1016/j.infbeh.2016.07.004.

44. Gronqvist $\mathrm{H}$, Brodd K, Rosander K. Development of smooth pursuit eye movements in very prematurely born infants: 2 . The low-risk subgroup. Acta Paediatricia. 201 I;100(7):5-1 I. doi: https://doi.org//0.1 I I //j. I65 I-2227.201 I.02247.x.

45. McConnell D, Parakkal M, Savage A, Rempel G. Parent-mediated intervention: adherence and adverse effects. Disability and Rehabilitation. 2014;37(10):1-9. doi: https://doi.org/10.3109/09638288.2014.946157.

46. Barbosa C, Balp M, Kulich K, Germain N, Rofail D. A literature review to explore the link between treatment satisfaction and adherence , compliance and persistence. Patient Preference and Adherence. 2012;6:39-48. doi: https://dx.doi.org/I0.2 147\%2FPPA.S24752.

47. Rone-Adams S, Stern D, Walker V. Stress and compliance with a home exercise program among caregivers of children with disabilities. Pediatric Physical Therapy. 2004; 16(3): 140-8. doi: https://doi.org/10.1097/0I.PEP.0000 136006.13449.DC.

48. Obeidat $\mathrm{H}$, Bond $\mathrm{E}$, Clark $\mathrm{C}$. The parental experience of having an infant in the newborn intensive care unit. The Journal of Perinatal Education. 2009; 18(3):23-9. doi: https://dx.doi.org/I0.1624\%2FI058|2409X46II99.

49. Young R. The importance of bonding. International Journal of Childbirth Education. 2013;28(3): I I-6.

50. Perszyk DR, Waxman SR. Infants' advances in speech perception shape their earliest links between language and cognition. Scientific reports. 2019;9(I): I-6. doi: https://doi.org/I0.1038/s4I598-0I9-395। I-9.

Corresponding Author:

* Megan Campbell

Email:megan.perrie@gmail.com 